\title{
USO DE AÇÚCARES EM PRODUTOS PANIFICADOS ${ }^{1}$
}

\author{
Mauricio Sergio ESTELLER², Rosa Maria de Oliveira YOSHIMOTO², \\ Renata Lira AMARAL ${ }^{2}$, Suzana Caetano da Silva LANNES $2, *$
}

\section{RESUMO}

Pão é um produto popular consumido na forma de lanches ou com refeições e apreciado devido a sua aparência, aroma, sabor, preço e disponibilidade. Depois de produzidos, os pães sofrem transformações que levam rapidamente à perda de crocância e ao endurecimento. Este processo tem impacto econômico grande, obrigando os produtores a trabalharem com data de validade curta, promoções, retorno de produtos não comercializados e aumento nos custos de produção e distribuição. A maioria dos produtos panificados apresenta algum tipo de açúcar que, além do enriquecimento calórico, funciona como melhorador de sabor, cor, plasticidade, conservação e auxiliar na fermentação. Neste estudo utilizou-se formulação padronizada, substituindo-se a sacarose da formulação inicial por outros açúcares (frutose líquida e cristalina, dextrose anidra, mel e açúcar invertido), mantendo-se o teor de sólidos totais e o de água. O tempo e a temperatura de assamento foram fixados em $8 \mathrm{~min}$ e $225^{\circ} \mathrm{C}$. Foram elaboradas análises de textura (dureza), umidade, volume, sensorial e vida-de-prateleira. Os resultados mostraram que a utilização de açúcares com características umectantes, se utilizados em quantidade adequada à formulação, podem melhorar a maciez e prolongar a vida-de-prateleira dos pães.

Palavras-chave: reologia; textura; açúcares; panificação.

\section{SUMMARY}

SUGAR EFFECT ON BAKERY PRODUCTS. Bread is a popular product consumed as a sandwich or during mealtime and appreciated by its appearance, flavor, taste, price and availability. After its production take place changes that leads to loss of crust crispness and increase of crumb firmness. Staling has a significant economic impact that lead the manufacturers to reduce the shelf-life, increase store discounts, production and distribution costs. The majority of bakery products contain some type of sugar which as font of energy improve flavor, color, softness, conservation and fermentation aid. A control bread formula was used changing sucrose from the original formula to different sweeteners (syrup and dry fructose, anhydrous dextrose, honey and inverted sugar), keeping the sweetness, and the amounts of total solids and water. The time and temperature of the oven were fixed in 8 minutes and $225^{\circ} \mathrm{C}$. Texture (firmness), moisture, volume, sensory analysis and shelf-life were evaluated. The results showed that it can be used in bread production different kinds of sugars alone or in combination to improve softness and shelf life of bread.

Keywords: rheology; texture; sweeteners; bakery products.

\section{1 - INTRODUÇÃO}

O pão é um produto bastante popular no Brasil, com consumo per capita de 27 quilos por ano [1], consumido na forma de lanche, ou mesmo junto com as refeições principais. A popularidade do pão é devida, sem dúvida, ao excelente sabor, preço e disponibilidade junto às milhares de padarias e supermercados do País. Crescendo rapidamente, o mercado de pães artesanais e industriais demanda a criação de novas padarias e fábricas, desenvolvimento de maquinário, receitas, aditivos e coadjuvantes. Grande parte do consumo brasileiro é representado pela linha constituída por pães com crosta fina - ou nenhuma - e bastante miolo. Pães de forma, hambúrguer, hot-dog, bisnagas - maioria impulsionada pelas cadeias de fast-food-além de pães especiais como dinner-roll e bisnaguinhas são bastante populares no café da manhã de hotéis, merenda escolar e companhias de aviação. O sucesso destes pães é caracteri-

\footnotetext{
1. Recebido para publicação em 10/03/2003. Aceito para publicação em 20/08/2004 (001077).

2. Departamento de Tecnologia Bioquímico-Farmacêutica-Faculdade de Ciências Farmacêuticas - USP, Av. Dr.Lineu Prestes, 580 - Bloco 16 CEP 05508-900 - São Paulo-SP. E-mail: esteller@usp.br / scslan@usp.br 3. Getec Guanabara Química Industrial S/A. Rua Flórida, 1737, $1^{\circ}$ andarCEP 04562-000. São Paulo-SP. E-mail: getecsp@uninet.com.br.

* A quem a correspondência deve ser enviada.
}

zado, sobretudo, pela maciez e facilidade de mastigação combinada com o uso de recheios cremosos como maionese, requeijão, margarinas, patês e outros.

Algumas horas depois de produzidos os pães sofrem transformações que levam rapidamente à perda de crocância (pão-francês, italiano, ciabatta) e endurecimento (pão para hot-dog, hambúrguer, pão de forma, bisnagas). Este processo é denominado de envelhecimento do pão ou bread staling [14, 26]. Isto ocorre devido à recristalização do amido, alterações das proteínas e migração da água da massa após a cocção [8, 9]. Este processo tem um impacto econômico bastante grande, o que obriga as empresas produtoras a trabalharem com data de validade (vida-de-prateleira) curta, traduzindo-se em um retorno grande de produtos não comercializados e aumento dos custos de produção e distribuição [28, 30].

TABELA 1. Açúcares utilizados em panificação [3].

\begin{tabular}{|c|c|c|c|c|c|c|c|c|}
\hline \multirow{2}{*}{ Tipo de açúcar } & \multicolumn{8}{|c|}{ Propriedades (\%) } \\
\hline & sólidos & água & poder dulçor $^{*}$ & sacarose & frutose & dextrose & maltose & outros \\
\hline mel & 81 & 17,2 & 97 & 1,31 & 38,2 & 31,3 & 7,3 & 1,5 \\
\hline açúcar-de-cana & 100 & - & 100 & 100 & - & - & - & - \\
\hline açúcar invertido (a) & 77 & 23 & 110 & 46 & 25 & 27 & - & 2 \\
\hline açúcar invertido (b) & 77 & 23 & 120 & 3 & 45 & 48 & - & 4 \\
\hline dextrose anidra & 91 & 9 & 75 & - & - & 100 & - & - \\
\hline xarope glicose $42 \mathrm{DE}$ & 80 & 20 & 50 & - & - & 19 & 14 & 67 \\
\hline frutose cristalina & 99 & 1 & 170 & - & 100 & - & - & - \\
\hline xarope frutose (HFS) & 80 & 20 & 140 & - & 86 & 13,5 & - & - \\
\hline
\end{tabular}

(a) inversão parcial; (b) inversão total

\% em relaçãoo à sacarose* 
Os açúcares, na formulação de pães têm sido utilizados, tradicionalmente, para o adoçamento, aumento de maciez e volume, desenvolvimento do sabor e cor [2] (Tabela 1).

- Mel - Um desenho em caverna na Espanha, datado de 7000 a.C, mostra um homem colhendo mel, o mesmo ocorrendo com registros egípcios de 5500 a.C. É conhecida há muito tempo a adição de mel em pães, sendo o único açúcar que não necessita de qualquer tipo de refino e pode ser utilizado, tal como produzido na natureza. Industrialmente, tem uso restrito devido às oscilações de preço e dificuldade de obtenção do produto padronizado. Apresenta variações de cor, sabor, umidade e conteúdo de açúcares [31, 33].

- Açúcar de cana - Alguns registros dão conta de plantações de cana feitas pelos aborígines da Nova Guiné 6000 a.C e produção de açúcar na Índia no século I. No século VII d.C., os árabes lançaramse à conquista de um grande império e, à medida que avançavam nas suas conquistas foram cultivando a cana de açúcar no Egito, na Palestina e no Norte da África. O açúcar chegou à Europa no século XI. Em Portugal, a cana foi cultivada no Algarves e na região de Coimbra desde o século XIV. Daí, passou para a ilha da Madeira, em meados do século seguinte [19].

- Açúcar Invertido - Cerca de 20\% mais doce que o açúcar de cana, o açúcar invertido é preparado pela hidrólise ácida ou enzimática da sacarose resultando em uma mistura de frutose e glicose.

- Dextrose anidra - Obtida pela hidrólise total do amido ou isolada a partir de hidrolisados de sacarose. Na forma cristalina apresenta $100 \%$ de concentração em dextrose.

- Frutose - O avanço no processo de manufatura da frutose tornou viável economicamente sua comercialização a partir da década de 60 [7]. Em escala industrial foram desenvolvidas tecnologias para obtenção de xaropes a partir do milho - HFS (highfructose syrups). Produzidos em concentrações de 42, 55 e 90\% em frutose, apresentam poder dulçor diferente em relação à sacarose. A maior parte dos HFS são produzidos e consumidos nos Estados Unidos [34].

No Brasil, o xarope de frutose é obtido através da hidrólise da sacarose e concentrado a $86 \%$ em frutose [10]. A frutose foi rapidamente integrada ao mercado de bebidas, panificação, conservas, geléias e laticínios [23]. Na forma cristalina é utilizada pela indústria farmacêutica, pré-misturas de bolos e produtos especiais [12, $25,29]$.

Dentre outros fatores, a retenção da água no produto final é de fundamental importância para a manutenção da maciez dos pães. Observa-se que isto ocorre devido à formação de ligações químicas entre as moléculas de água e as moléculas dos açúcares. Esta atração é suficiente para prevenir a migração e a eva- poração da água, mantendo-a ligada mesmo durante o forneamento.

Inicialmente, no forno a $225^{\circ} \mathrm{C}$, ocorre a evaporação da água de condensação formada na câmara de fermentação (crescimento final), a volatilização de compostos orgânicos e etanol, a expansão do gás carbônico e vapor d'água do miolo, resultando em grande expansão da massa [13].

Com o aumento sucessivo da temperatura interna, até $98^{\circ} \mathrm{C}$, ocorre a inativação do fermento e das enzimas, a gelatinização do amido (amilose e amilopectina) e modificações das proteínas (glutenina e gliadina). Finalmente, uma crosta fina é formada com o aparecimento da cor castanho-dourada característica, em função da caramelização dos açúcares e da reação de Maillard [17], cujo mecanismo simplificado pode ser descrito como:

Açúcar redutor + amino-composto $\rightarrow$ glicosilaminas $\rightarrow$ melanoidinas (castanho-dourado)

A reologia é o estudo da mecânica da deformação permanente ou temporária dos materiais sólidos e líquidos. No processamento, os alimentos são submetidos a forças como tensão e cisalhamento durante as operações de redução de tamanho, prensagem, extrusão, aquecimento e resfriamento [5]. A textura pode ser definida como todos os atributos mecânicos, geométricos e de superfície de um produto que sejam perceptiveis por meios instrumentais e sensoriais [27]. O procedimento para determinação da dureza, um dos parâmetros de textura, consiste em submeter os pães à compressão, e da análise da curva força-tempo resultante, toma-se como parâmetro a força máxima aplicada às amostras [20, 21, 24].

A diminuição no sabor, aroma e ressecamento dos pães podem ser avaliados por painel treinado, mas os consumidores, em geral, comprimem o pão (squeeze test) para avaliar sua maciez. A resistência avaliada por compressímetros mecânicos correlacionam de forma adequada esta característica [32].

Este trabalho objetiva avaliar a influência da adição de diferentes açúcares em pão para hambúrguer, em substituição total ou parcial, enfatizando as características reológicas [16, 27].

\section{2 - MATERIAL E MÉTODOS}

\section{1 - Material}

Foi utilizada farinha de trigo especial (Sol), adquirida em um único lote e armazenada em câmara fria a $-18^{\circ} \mathrm{C}$, apresentando $14 \%$ umidade, glúten úmido $30 \%$ e $0,5 \%$ cinzas máx (de acordo com laudo de análise do fabricante); fermento biológico fresco prensado (Itaiquara), armazenado em geladeira a $7^{\circ} \mathrm{C}$; sal refinado de mesa (Cisne); açúcar refinado (União); gordura vegetal hidrogenada tipo Mesa $\mathrm{H}$ (Refino de Óleos Brasil); enzima alfa-amilase fúngica A4000 Grindamyl (Danisco), diluída com malto-dextrina 1:1000; propio- 
nato de cálcio (Krishna Organic); mel de abelha de flores silvestres (Apiário do Instituto de Biociências USP); xarope de frutose concentração $86 \%$ em frutose, base seca (Getec); frutose cristalina anidra 100\% frutose (Danisco); açúcar invertido 77\% sólidos (Dulcini); dextrose anidra $100 \%$ (Getec).

\section{2 - Preparo das amostras}

As massas foram preparadas pelo sistema de massa direta [22], onde todos os ingredientes são misturados de uma só vez [15], sem pré-fermentação (esponja), em batedeira planetária KitchenAid 525W/120V/ $60 \mathrm{~Hz}$ (Brastemp). Os pães foram assados em forno elétrico tipo estacionário 4000W/220V (Flexa de Ouro, SP). As formulações e processo empregados são apresentados, respectivamente, na Tabela 2 e Figura 1.

TABELA 2. Formulações utilizadas para o teste experimental de panificação.

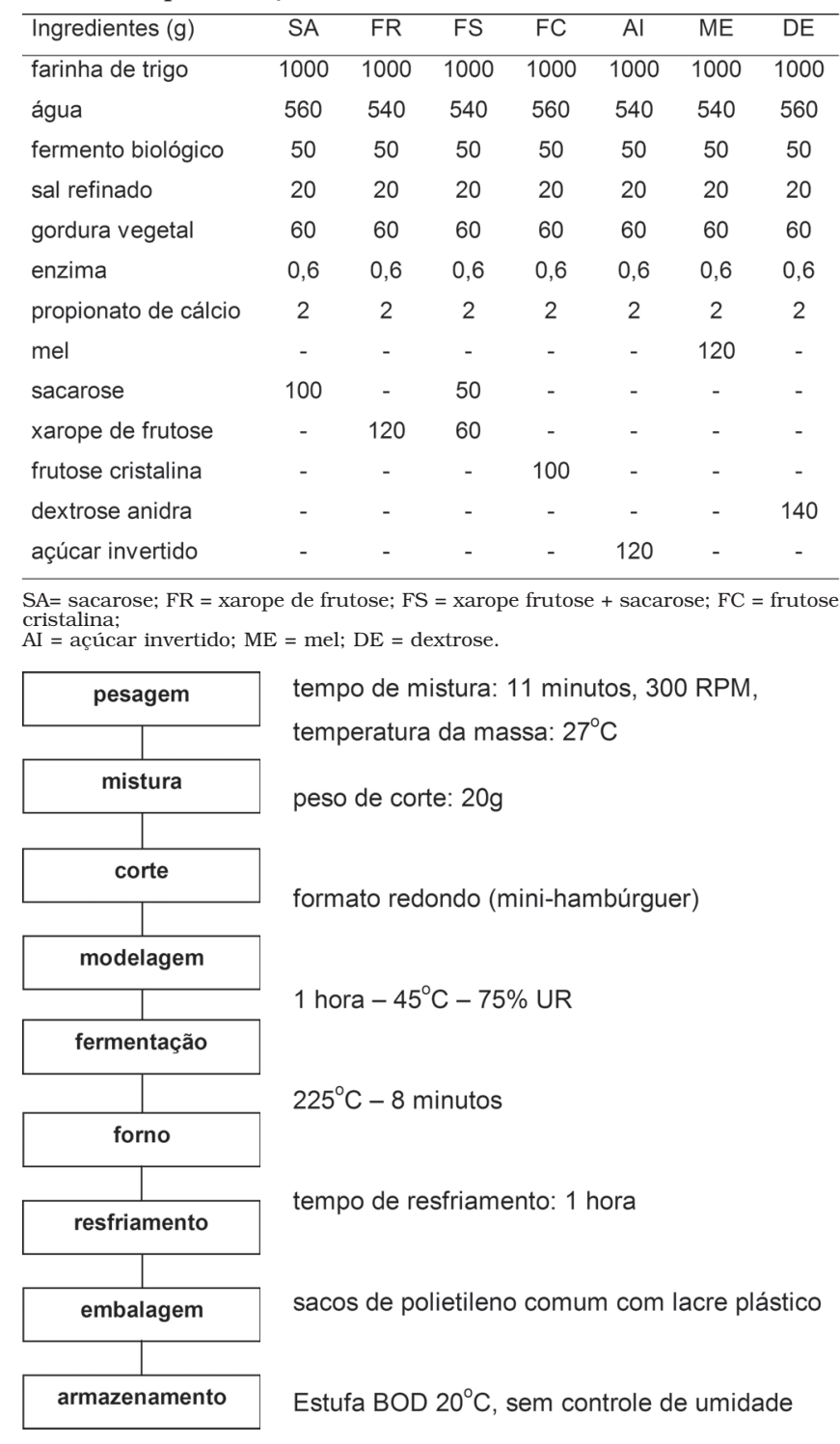

FIGURA 1. Fluxograma utilizado para o teste de panificação

\section{3 - Análise sensorial}

Foi realizado um teste de aceitação para pães com um dia de armazenagem, preparados com xarope de frutose (FR), sacarose (SA) e combinação de frutose com sacarose (FS), considerando os atributos sabor, maciez e aspecto geral. Utilizou-se escala hedônica estruturada de 9 pontos [11, 21]. As amostras foram servidas com apresentação monádica seguindo delineamento completo de blocos casualizados, com 46 consumidores locais (70\% mulheres), consumidores habituais deste tipo de produto, com idades de 17 a 45 anos. Os dados, coletados em fichas (Figura 2), foram submetidos à distribuição de freqüência e análise de variância (ANOVA) com fator duplo amostra/provador.

\section{4 - Análise de umidade}

Para a análise de umidade o pão foi picado e homogeneizado (casca+miolo) pesando-se $10 \mathrm{~g}$ da amostra em cápsula de alumínio tarada [18].

\section{5 - Análise de textura}

Todas as formulações foram produzidas em triplicata, com leituras iniciadas no 2 ㅇ dia de armazenamento (para redistribuição da umidade na massa), durante 10 dias consecutivos, tempo de prateleira para este tipo de produto. Foi utilizado o texturômetro TA.XT2 - Stable Micro Systems nos seguintes parâmetros: probe compression platens $\mathrm{P} / 100 \phi 100 \mathrm{~mm}$, dupla compressão $2,0 \mathrm{~mm} / \mathrm{s}$, distância $40 \%$, teste de ruptura $1,0 \%$, força $100 \mathrm{~g}$, tempo $5 \mathrm{~s}$. Tomou-se o parâmetro de dureza para a avaliação dos resultados, que representam a média de dez leituras de cada formulação.

\section{6 - Análise do volume}

O volume dos pães foi determinado, em triplicata, colocando-se as amostras em copo de vidro com volume pré-determinado, completando-se os espaços com micro-esferas de polietileno de densidade conhecida e leitura remanescente em cilindro graduado expressando-se o resultado em $\mathrm{cm}^{3}$. 


\section{7 - Vida-de-prateleira (shelf-life)}

Os pães foram acondicionados em sacos plásticos de polietileno comum e armazenados em estufa $\mathrm{BOD}$ a $20^{\circ} \mathrm{C}$, com o acompanhamento de crescimento fúngico. Sua validade foi anotada e descartada ao primeiro sinal de aparecimento de colônias cotonosas de cores variadas $[4,6]$.

\section{8 - Análise dos dados}

Para tratamento estatístico dos dados foi utilizado o software Statistica 6.0/2001 (StatSoft, Inc. Tulsa, OK, EUA).

\section{3 - RESULTADOS E DISCUSSÃO}

\section{1. - Textura e umidade}

Os resultados das análises de textura (dureza) e umidade são apresentados em função dos dias de armazenamento nas Tabelas 3 e 4 . Para o parâmetro de dureza, observou-se para todas as formulações que houve acréscimo com o decorrer dos dias, com variações dos resultados, podendo-se justificar pelas diferentes formas de recristalização do amido, alterações das proteínas e migração da água na massa após a cocção [26]. Embora nos dois primeiros dias, a amostra com FC tenha se mostrado como uma das mais duras, a perda de sua maciez ocorreu mais lentamente que as demais amostras. No final da avaliação, os pães com FC somente apresentaram-se mais duros que os pães com açúcar invertido e dextrose sendo que estas amostras apresentaram-se como as mais macias desde o início.

$\mathrm{Na}$ comparação dos resultados obtidos para SA e FS, verificou-se que a substituição parcial da sacarose por xarope de frutose conferiu maior dureza ao pão, embora nos 3 primeiros dias os pães com ambos os açúcares estivessem mais macios que os pães com sacarose somente. No final da avaliação, a amostra com FS apresentou-se tão dura quanto a amostra com mel e ambas menos duras apenas que as amostras com xarope de frutose.

Para a umidade ocorreram variações nas diversas formulações, sendo que nos produtos elaborados com açúcares que possuem propriedades umectantes (xarope de frutose, frutose cristalina, açúcar invertido, dextrose) parece existir a tendência ao aumento de umidade com os dias de armazenamento.

O envelhecimento do pão (staling) não está relacionado com a perda de umidade da massa. Um pão analisado após 5 dias (armazenado em "condições apropriadas") possuiria a mesma umidade que um pão "fresco" embora apresente ao paladar a sensação de estar mais seco. O endurecimento estaria vinculado à retrogradação do amido e ao comportamento das proteínas e pentosanas como observado por STAUFFER [32].

Em estudos anteriores, a utilização de mel como agente umectante em pães de forma, em niveis de $3 \%$ e $6 \%$ em relação à farinha, não mostrou resultados significativos para tempo de fermentação, rendimento, cor da crosta e miolo, textura, aroma e sabor [33].
ASKAR et al. [2] estudaram o efeito do uso de misturas de sacarose e alguns tipos de substitutos de açúcares, entre eles a frutose, também na proporção 50:50, em bolos dietéticos. Encontraram que substituições de sacarose acima de $25 \%$ resultaram no decréscimo na qualidade e aceitabilidade dos bolos, sendo que para pães o mesmo não ocorreu.

No experimento, a adição do açúcar invertido, forneceu o melhor resultado nos quesitos maciez e umidade, mas de uma forma geral, os açúcares conhecidos por suas propriedades higroscópicas, não assumiram tal funcionalidade para o tipo de produto estudado, podendo ser devido à quantidade utilizada em cada formulação.

TABELA 3. Valores de dureza (gf) versus dias de armazenamento para todas as amostras.

\begin{tabular}{cccccccc}
\hline dias & \multicolumn{7}{c}{ formulações } \\
\hline & SA & ME & FS & FR & FC & Al & DE \\
\hline 1 & $1612,2^{\mathrm{e}}$ & $1413,8^{\mathrm{c}}$ & $1573,2^{\mathrm{d}}$ & $1886,7^{\mathrm{f}}$ & $1741,2^{\mathrm{b}}$ & $1193,1^{\mathrm{b}}$ & $1043,8^{\mathrm{a}}$ \\
2 & $2015,0^{\mathrm{d}}$ & $2009,9^{\mathrm{d}}$ & $1807,5^{\mathrm{c}}$ & $2610,3^{\mathrm{f}}$ & $2401,7^{\mathrm{e}}$ & $1793,8^{\mathrm{b}}$ & $1460,3^{\mathrm{a}}$ \\
3 & $2728,8^{\mathrm{d}}$ & $3114,0^{\mathrm{e}}$ & $2530,9^{\mathrm{c}}$ & $2457,9^{\mathrm{b}}$ & $2470,1^{\mathrm{b}}$ & $2436,5^{\mathrm{b}}$ & $2218,4^{\mathrm{a}}$ \\
4 & $2776,7^{\mathrm{c}}$ & $2657,3^{\mathrm{e}}$ & $3262,5^{\mathrm{d}}$ & $2749,0^{\mathrm{c}}$ & $2469,2^{\mathrm{a}}$ & $2537,3^{\mathrm{b}}$ & $2589,7^{\mathrm{b}}$ \\
5 & $2861,5^{\mathrm{b}}$ & $3633,8^{\mathrm{f}}$ & $3120,2^{\mathrm{d}}$ & $3214,5^{\mathrm{e}}$ & $2941,0^{\mathrm{c}}$ & $2915,5^{\mathrm{c}}$ & $2535,9^{\mathrm{a}}$ \\
6 & $2860,4^{\mathrm{b}}$ & $3718,5^{\mathrm{e}}$ & $3402,3^{\mathrm{d}}$ & $2994,8^{\mathrm{c}}$ & $3008,6^{\mathrm{c}}$ & $2624,7^{\mathrm{a}}$ & $2615,5^{\mathrm{a}}$ \\
7 & $3210,0^{\mathrm{b}}$ & $2949,6^{\mathrm{c}}$ & $3450,4^{\mathrm{d}}$ & $3114,6^{\mathrm{b}}$ & $3000,0^{\mathrm{a}}$ & $3096,7^{\mathrm{a}}$ & $3027,8^{\mathrm{a}}$ \\
8 & $3302,5^{\mathrm{c}}$ & $3376,4^{\mathrm{d}, \mathrm{e}}$ & $3700,3^{\mathrm{d}}$ & $3822,2^{\mathrm{e}}$ & $3006,2^{\mathrm{b}}$ & $2746,7^{\mathrm{a}}$ & $3050,8^{\mathrm{b}}$ \\
9 & $3604,3^{\mathrm{d}}$ & $3809,7^{\mathrm{e}}$ & $3830,8^{\mathrm{e}}$ & $4146,5^{\mathrm{f}}$ & $3101,0^{\mathrm{c}}$ & $2995,3^{\mathrm{a}}$ & $3029,0^{\mathrm{b}}$ \\
\hline
\end{tabular}

$\mathrm{SA}=$ sacarose; $\mathrm{FR}=$ xarope de frutose; $\mathrm{FS}=$ xarope frutose + sacarose; $\mathrm{FC}=$ frutose cristalina;

$\mathrm{AI}=$ açúcar invertido; $\mathrm{ME}=\mathrm{mel} ; \mathrm{DE}=$ dextrose

Lẽras ções que foram analisadas no mesmo dia $(\mathrm{p}<0,01)$ Tukey HSD.

TABELA 4. Valores de umidade (\%) versus dias de armazenamento para todas as amostras.

\begin{tabular}{cccccccc}
\hline dias & \multicolumn{7}{c}{ formulações } \\
\hline & $\mathrm{SA}$ & $\mathrm{ME}$ & $\mathrm{FS}$ & $\mathrm{FR}$ & $\mathrm{FC}$ & $\mathrm{Al}$ & $\mathrm{DE}$ \\
\hline 1 & $30,14^{\mathrm{a}}$ & $29,72^{\mathrm{a}}$ & $29,20^{\mathrm{a}}$ & $29,58^{\mathrm{a}}$ & $29,61^{\mathrm{a}}$ & $31,56^{\mathrm{o}}$ & $29,92^{\mathrm{a}}$ \\
2 & $28,98^{\mathrm{a}, \mathrm{b}}$ & $29,92^{\mathrm{c}, \mathrm{d}}$ & $30,11^{\mathrm{d}}$ & $29,78^{\mathrm{c}, \mathrm{d}}$ & $29,27^{\mathrm{b}, \mathrm{c}}$ & $28,16^{\mathrm{a}}$ & $28,86^{\mathrm{a}, \mathrm{b}}$ \\
3 & $30,90^{\mathrm{c}}$ & $28,07^{\mathrm{a}}$ & $31,00^{\mathrm{c}}$ & $29,48^{\mathrm{a}, \mathrm{b}}$ & $28,92^{\mathrm{a}, \mathrm{b}}$ & $29,49^{\mathrm{b}}$ & $29,80^{\mathrm{b}, \mathrm{c}}$ \\
4 & $30,22^{\mathrm{d}}$ & $27,22^{\mathrm{a}}$ & $29,34^{\mathrm{b}, \mathrm{c}, \mathrm{d}}$ & $28,67^{\mathrm{b}, \mathrm{c}}$ & $28,70^{\mathrm{a}, \mathrm{b}}$ & $30,01^{\mathrm{c}, \mathrm{d}}$ & $29,39^{\mathrm{b}, \mathrm{c}, \mathrm{d}}$ \\
5 & $29,90^{\mathrm{c}}$ & $27,27^{\mathrm{a}}$ & $28,46^{\mathrm{b}}$ & $28,45^{\mathrm{a}, \mathrm{b}}$ & $27,30^{\mathrm{a}}$ & $28,58^{\mathrm{b}}$ & $29,80^{\mathrm{c}}$ \\
6 & $29,76^{\mathrm{d}, \mathrm{e}}$ & $26,75^{\mathrm{a}}$ & $27,69^{\mathrm{b}}$ & $28,96^{\mathrm{c}, \mathrm{d}}$ & $28,50^{\mathrm{b}, \mathrm{c}}$ & $30,56^{\mathrm{e}}$ & $28,71^{\mathrm{c}}$ \\
7 & $29,50^{\mathrm{c}, \mathrm{d}}$ & $27,68^{\mathrm{a}}$ & $28,12^{\mathrm{a}, \mathrm{b}}$ & $29,05^{\mathrm{b}, \mathrm{c}}$ & $29,12^{\mathrm{b}, \mathrm{c}}$ & $30,40^{\mathrm{d}}$ & $30,40^{\mathrm{d}}$ \\
8 & $28,80^{\mathrm{a}, \mathrm{b}}$ & $27,51^{\mathrm{a}}$ & $28,50^{\mathrm{a}, \mathrm{b}}$ & $30,10^{\mathrm{c}}$ & $29,64^{\mathrm{b}, \mathrm{c}}$ & $30,30^{\mathrm{c}}$ & $30,30^{\mathrm{c}}$ \\
9 & $29,10^{\mathrm{b}, \mathrm{c}}$ & $27,22^{\mathrm{a}}$ & $28,60^{\mathrm{a}, \mathrm{b}}$ & $32,70^{\mathrm{c}}$ & $30,41^{\mathrm{c}}$ & $29,90^{\mathrm{c}}$ & $29,80^{\mathrm{b}, \mathrm{c}}$
\end{tabular}

SA= sacar

cristalina;
$\mathrm{AI}=$ açúcar invertido; $\mathrm{ME}=\mathrm{mel} ; \mathrm{DE}=$ dextrose
Letras iguais na mesma linha indicam variacão não significativa entre as formulações analisadas no mesmo dia ( $p<0,01)$ Tukey HSD.

\section{2 - Análise sensorial}

$\mathrm{Na}$ análise estatística para sabor, obteve-se um $\mathrm{F}_{\text {calc }}$ $(7,068477)$ maior que o $\mathrm{F}_{\text {tab }}(4,765866)$, indicando que as amostras são significativamente diferentes entre si a $5 \%$ de nível de significância. Sendo que, nas observações dos provadores, encontrou-se menção a diferenças maiores no pão com xarope de frutose e preferência pela sacarose.

A frutose anidra apresenta poder dulçor 1,5 a 1,7 vezes maior que a sacarose cristalina. O pão com xarope 
de frutose (FR), apresentou-se ligeiramente mais adocicado aos provadores e detectado na análise estatística.

Para a maciez: o $F_{\text {calc }}(4,013322)$ é menor que $F_{\text {tab }}$ $(4,765866)$, e as amostras não são significativamente diferentes entre si a 5\% de nível de significância.

O teor de açúcares empregado (10\% em relação à farinha de trigo) não exerceu influência significativa na maciez para uma detecção significativa por meios sensoriais, porém tendo sido detectado no texturômetro.

Já no aspecto geral o $F_{\text {calc }}(2,403572)$ é menor que $\mathrm{F}_{\text {tab }}(4,765866)$, portanto amostras não são significativamente diferentes entre si a $5 \%$ de nível de significância.

\section{3 - Volume}

O tipo de açúcar utilizado, não influenciou de forma significativa a fermentação, retenção de gases e volume final do produto. Apesar de estatisticamente diferentes $(\mathrm{p}<0,01)$, variações em até $5 \%$ em relação à média são aceitas pelos fabricantes, uma vez que outras variáveis como a quantidade de fermento, teor de proteína na farinha, agentes oxidantes, peso de corte e processo podem influenciar de forma muito mais marcante o volume final (Tabela 5).

TABELA 5. Valores de volume $\left(\mathrm{cm}^{3}\right)$ para todas as formulações

\begin{tabular}{cc}
\hline Formulações & Volume $\left(\mathrm{cm}^{3}\right)$ \\
\hline SA & $64^{\mathrm{b}}$ \\
ME & $62^{\mathrm{a}, \mathrm{b}}$ \\
FS & $64^{\mathrm{b}}$ \\
FR & $60^{\mathrm{a}}$ \\
FC & $63^{\mathrm{b}}$ \\
Al & $62^{\mathrm{b}}$ \\
DE & $63^{\mathrm{b}}$ \\
\hline
\end{tabular}

$\mathrm{SA}=$ sacarose; $\mathrm{FR}=$ xarope de frutose;

$\mathrm{FS}=$ xarope frutose + sacarose; $\mathrm{FC}=$ frutose cristalina

$\mathrm{AI}=$ açúcar invertido; $\mathrm{ME}=$ mel; $\mathrm{DE}=$ dextrose

Letras iguais indicam variação não significativa entre as formulações $(p<0,01)$ Tukey HSD.

\section{4 - Vida-de-prateleira (shelf-life)}

O tipo de açúcar para as formulações propostas não exerceu influência, sendo que a maioria das amostras apresentou vida-de-prateleira de 9 a 10 dias. A Tabela 6 apresenta o aparecimento de bolor nos pães indicados por (+).

TABELA 6. Vida-de-prateleira (dias) das amostras.

\begin{tabular}{|c|c|c|c|c|c|c|c|}
\hline Dias & & & & רostr & & & \\
\hline & $\mathrm{SA}$ & $\mathrm{ME}$ & FS & 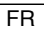 & FC & $\overline{\mathrm{Al}}$ & $\mathrm{DE}$ \\
\hline 1 & - & - & - & - & - & - & - \\
\hline 2 & - & - & - & - & - & - & - \\
\hline 3 & - & - & - & - & - & - & - \\
\hline 4 & - & - & - & - & - & - & - \\
\hline 5 & - & - & - & - & - & - & - \\
\hline 6 & - & - & - & - & - & - & - \\
\hline 7 & - & - & - & - & - & - & - \\
\hline 8 & - & - & - & - & + & - & - \\
\hline 9 & + & + & - & - & + & - & + \\
\hline 10 & + & + & + & + & + & + & + \\
\hline
\end{tabular}

\section{4 - CONCLUSÕES}

A interação entre os ingredientes é fator primordial para a obtenção de um produto de boa qualidade e que deverá ser considerada para um ajuste de formulação. A análise instrumental detectou variações que não foram percebidas sensorialmente. Portanto, no tipo de produto estudado, a utilização de açúcares com propriedades umectantes se utilizados em quantidade adequada à formulação, tendem a melhorar a maciez dos pães e prolongar a vida-de-prateleira.

\section{5- REFERÊNCIAS BIBLIOGRÁFICAS}

[1] ASSOCIAÇÃO BRASILEIRA DAS INDÚSTRIAS DE PANIFICAÇÃO E CONFEITARIA. Análise do mercado de pães, 2002. Disponível em: http://www.abip.org.br. Acesso em: 10 jun. 2002.

[2] AIKAR, A.; ABDEL-FADEEL, M.G.; MONA, A.; E1RAKAYBI, A.M.A.; MOSTAFA, G.A. Studies on the introduction of dietetic cake using sweeteners as sugar substitute. Dtsch. Lebensm.-Rundsch., Stuttgart, v. 83, p. 389, 1993.

[3] BAKER'S DIGEST. Sweeteners for baking, p. 35,v. 33, 1986.

[4] BEUCHAT, L.R. Microbial stability as affected by water activity. Cereal Foods World, St. Paul, v. 26, n. 7, p. 345-349, 1981.

[5] BRASEQ. Parâmetros físicos relevantes ao processamento de alimentos. Food Ingredients, São Paulo, n. 10, p. 94-95, 2001. [Guia de fornecedores].

[6] BUERA, M.P.; JOUPPILA, K.; CHIRIFE, J. Differential scanning calorimetry glass transition temperatures of white bread and mold growth in the putative glassy state. Cereal Chem., St. Paul, v. 75, n. 1, p. 64-69, 1998.

[7] CASEY, J.P. High fructose corn syrup: a case of innovation. Cereal Foods World, St. Paul, v. 22, n. 2, p. 49-55, 1977.

[8] CZUCHAJOWSKA, Z. POMERANZ, Y. Differential scanning calorimetry, water activity, and moisture contents in crumb center and near-crust zones of bread during storage. Cereal Chem., St. Paul, v. 66, n. 4, p. 305-309, 1989.

[9] FESSAS, D.; SCHIRALDI, A. Texture and staling of wheat bread crumb: effects of water extractable proteins and 'pentosans'. Thermochim. Acta, Amsterdam, v. 323, n. 1/2, p. 17-26, 1998.

[10] GETEC Guanabara Guímica. Xarope de frutose. Resende: GETEC, 2002. 2p. [Boletim técnico].

[11] GUTKOSKI, L.C.; PAVANELLI, A.P.; MIRANDA, M.Z.; CHANG, Y.K. Efeito de melhoradores nas propriedades reológicas e de panificação da massa de farinha de trigo. Ciênc. Tecnol. Aliment., Campinas, v. 17, n. 1, p. 11-16, 1997.

[12] HANOVER, L.M.; WHITE, J.S. Manufcturing, composition, and applications of fructose. Am. J. Clin. Nutr., Bethesda, n. 58, p. 724-732, 1993.

[13] HAYMAN, D.; HOSENEY, R.C.; FAUBION; J.M. Bread crumb grain development during baking. Cereal Chem., St. Paul, v. 75, n. 5, p. 577-580, 1998.

[14] HE H., HOSENEY, R.C. Changes in bread and moisture during long-term storage. Cereal Chem., St. Paul, v. 67, n. 6, p. 603-605, 1990. 
[15] HOSENEY, R.C.; SMEWING, J. Instrumental measurement of stickiness of doughs and other foods. J. Texture Stud., Trumbull, v. 30, p. 123-136, 1999.

[16] HOSENEY, R.C. Principles of cereal science and technology. American Association of Cereal Chemists, St. Paul, Minnesota, USA, p. 400-456, 1994, 825 p.

[17] HUG-ITEN, S.; ESCHER, F.; CONDE-PETIT, B. Structural properties of starch in bread and bread model systems: Influence of an antistaling a-amylase. Cereal Chem., St. Paul, v. 78, n. 4, p. 421-428, 2001.

[18] INSTITUTO ADOLFO LUTZ. Normas analíticas do Instituto Adolfo Lutz: métodos químicos e físicos para análise de alimentos. São Paulo: IAL, 1976. v. 1, p. 371.

[19] JAMES, C.P.C.; CHUNG, C.C. Cane sugar handbook: a manual for cane sugar manufacturers and their chemists. 11.ed., Hoboken, Hardcover, 1993. 1120p.

[20] LANNES, S.C.S.; GIOIELLI, L.A. Consistência de manteiga de cacau e seus sucedâneos comerciais: análise comparativa entre penetrômetro de cone e analisador de textura. In: CONGRESO Y EXPOSICIÓN LATINOAMERICANO SOBRE PROCESSAMENTO DE GRASAS Y ACEITES, Campinas, 1995. Anais. Campinas: UNICAMP, 1995. p. 229-233.

[21] LAWLESS, H.T.; HEYMANN, H. Sensory evaluation of food: principles and practices. New York: Chapman \& Hall, 1998. 820p.

[22] LORENZ, K.J.; KULP, K. Handbook of cereal science and technology. New York: Marcel Dekker, 1991. p. 639-679.

[23] MEINCKE, K. Fructose the natural sweetener with many advantages and characteristics. Confectionary Produc., Oxford, v. 52, n. 8, p. 519-521, 1986.

[24] MINIM, V.P.R.; MACHADO, P.T.; CANAVESI, E.; PIROZI, M.R. Perfil sensorial e aceitabilidade de dife- rentes formulações de pão de queijo. Ciênc. Tecnol. Aliment., Campinas, v. 20 n. 2, p. 154-159, 2000.

[25] OSBERGER, T.F.; OLINGER, P.M. Fructose gives desserts a glycemic advantage. Baker's Digest, Merrian, maio, 1985. p. 30-32.

[26] OVADIA, D.Z.; WALKER, C.E. Re-examination of the bread firming curve. Starch/Staerke, Weinheim, v. 48, n. 4, p. 137-144, 1996.

[27] ROSENTHAL, A.J. Food texture: measurement and perception. London: Chapman \& Hall, 1999. 311 p.

[28] SAHLSTROM, S.; BRATHEN, E. Effects of enzyme preparations for baking, mixing time and resting time on bread quality and bread staling. Food Chem., Amsterdam, v. 58, n. 1/2, p. 75-80, 1997.

[29] SAUSSELE Jr., H.; ZIEGLER, H.F.; WEIDEMAN, J.H. High fructose corn syrups for bakery applications. Baker's Digest, Merrian, jul./aug. 1983. p. 26-28.

[30] SI, J.Q. Synergistic effect of enzymes for breadbaking. Cereal Foods World, St. Paul, v. 42, n. 10, p. 802-807, 1997.

[31] SQUIRES, N.K.; D'ARCY, B.R.; CAFFIN, N.A. Australian honey as a ingredient in white pan bread to retard staling. In: AUSTRALIAN CEREAL CHEMISTRY CONFERENCE, Melbourne, 1997, p. 42-46.

[32] STAUFFER, C.E. Emulsifiers as antistaling agents. Cereal Foods World, St. Paul, v. 45, n. 3, p. 106-110, 2000 .

[33] STRAIT, M.J. The effect of liquid or dry honey as a partial replacement for sugar on the baking and keeping qualities of fat reduced muffins. Blacksburg, 1997. 175p. Thesis - Faculty of the Virginia Polytechnic Institute and State University.

[34] VUILLEUMIER, S. Worldwide production of highfructose syrup and crystalline fructose. Am. J. Clin. Nutr., Bethesda, n. 58, p. 115-118, 1993. 